\title{
Nanoscale perpendicular magnetic island arrays fabricated by extreme ultraviolet interference lithography
}

\author{
F. Luo, ${ }^{1, a)}$ L. J. Heyderman, ${ }^{1}$ H. H. Solak, ${ }^{1}$ T. Thomson, ${ }^{2, b)}$ and M. E. Best ${ }^{2}$ \\ ${ }^{1}$ Laboratory for Micro- and Nanotechnology, Paul Scherrer Institut, CH-5232 Villigen PSI, Switzerland \\ ${ }^{2}$ Hitachi Global Storage Technologies, San Jose Research Center, San Jose, California 95135, \\ USA
}

(Received 28 November 2007; accepted 20 January 2008; published online 11 March 2008)

\begin{abstract}
Magnetic island arrays with a period of $50 \mathrm{~nm}$ and uniform over $20 \times 20 \mu \mathrm{m}^{2}$ have been fabricated by depositing $\mathrm{Co} / \mathrm{Pd}$ multilayer films on prepatterned $\mathrm{SiO}_{x}$ pillars produced by extreme ultraviolet interference lithography. Scanning electron microscopy and magnetic force microscopy measurements made on the same islands give a direct, island-by-island comparison of the size and remanent switching field. The results demonstrate that the switching field distribution (SFD) is not primarily due to magnetostatic interactions, and a strong dependence of SFD on size is also not observed, indicating that a distribution of material properties is likely to be responsible for the SFD. (C) 2008 American Institute of Physics. [DOI: 10.1063/1.2841821]
\end{abstract}

The fabrication of large arrays of nanoscale magnetic islands not only gives the possibility to study magnetism at length scales approaching the exchange length ${ }^{1}$ but is also critical in the race to increase the density of magnetic data storage. ${ }^{2}$ Areal density growth in current granular magnetic media materials is limited by the appearance of superparamagnetism, ${ }^{3}$ which occurs when the grain volume is too small to prevent thermal fluctuations from spontaneously reversing the grain magnetization direction. By patterning the magnetic recording media into isolated small elements, referred to as bit patterned media (BPM), the onset of the superparamagnetic effect can be delayed. ${ }^{4,5}$ Here, each bit, rather than each grain, is a single magnetic switching volume and therefore provides greater thermal stability. If BPM is to replace current continuous media, a narrow switching field distribution (SFD) is required, $(\sigma /<$ mean $>$ less than $10 \%$ ), so that during recording only the intended bit is written, leaving the adjacent bits undisturbed. ${ }^{6}$ In BPM, the broadening of the SFD is attributed to several causes, including the lithographic irregularities, ${ }^{7}$ the distribution of the intrinsic magnetic anisotropy, ${ }^{8}$ and the magnetostatic interaction of the neighboring islands. ${ }^{9}$ In order to determine the viability of BPM for future magnetic recording, it is necessary to determine which factors give the most important contributions to the SFD.

Top down methods employed to pattern magnetic films include electron beam lithography, focused ion beam lithography, optical interference lithography, and nanoimprint lithography. ${ }^{2}$ In addition, several methods make use of natural ordering processes, such as self-assembly of magnetic nanoparticles, and phase separation in diblock copolymers used as etch masks or in a lift-off process to create magnetic islands. ${ }^{2}$ The fabrication technique employed in this work is extreme ultraviolet interference lithography (EUV-IL) based on EUV exposure through a mask consisting of diffraction gratings to form interfering beams. ${ }^{10,11}$ This technique has the advantage that it not only provides high resolution, with periods down to a theoretical limit of $6.5 \mathrm{~nm}$ (half the EUV

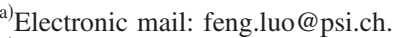

${ }^{b}$ Present address: School of Computer Science, University of Manchester, Manchester M13 9PL, UK.
}

wavelength) and precise positioning of the islands, but also provides a high throughput (with parallel processing and exposure times in the 5-30 s range) and can expose large areas up to several millimeters, depending on the structure size. ${ }^{11}$

In this letter, we report on the fabrication and switching properties of $50 \mathrm{~nm}$-period arrays of islands with perpendicular magnetic anisotropy made by depositing $\mathrm{Co} / \mathrm{Pd}$ multilayer films on substrates prepatterned with $\mathrm{SiO}_{x}$ pillars fabricated with EUV-IL. The use of prepatterned substrates allows the fabrication of magnetic nanostructures from any material that can be deposited as a thin film. Since the deposition of the magnetic film is the last step of the process, any possible degradation of the magnetic properties due to subsequent processing is avoided. This is critical for very small magnetic structures and prevents further broadening of the SFD. Co/Pd multilayers ${ }^{12}$ are excellent candidate materials for BPM since they exhibit a number of desired properties; they are highly exchange coupled promoting the formation of single domain islands, they have a large and controllable perpendicular magnetic anisotropy (providing a high thermal stability), and they have a sufficient saturation magnetization to allow reading of the direction of magnetization. In this work, we investigate the magnetic switching behavior of $\mathrm{Co} / \mathrm{Pd}$ multilayer islands with diameters of less than $30 \mathrm{~nm}$, extending the current knowledge about these materials ${ }^{8}$ down to smaller sizes.

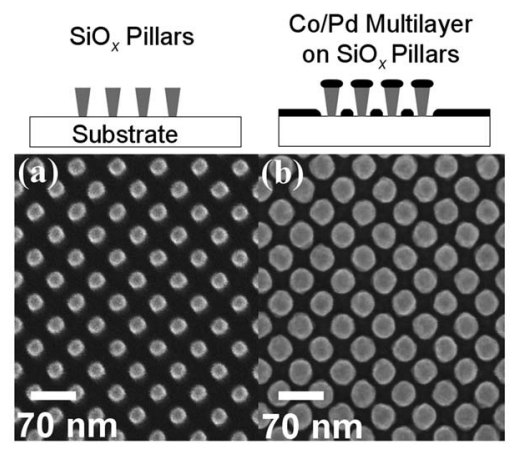

FIG. 1. Schematic diagram of the fabrication of nanoscale magnetic island arrays with EUV-IL and corresponding SEM images of $\mathrm{SiO}_{x}$ pillars with periods of $50 \mathrm{~nm}$ (a) before and (b) after $\mathrm{Co} / \mathrm{Pd}$ multilayer film deposition $\left[d_{\mathrm{tot}}=21.5 \mathrm{~nm}(n=15)\right]$. 


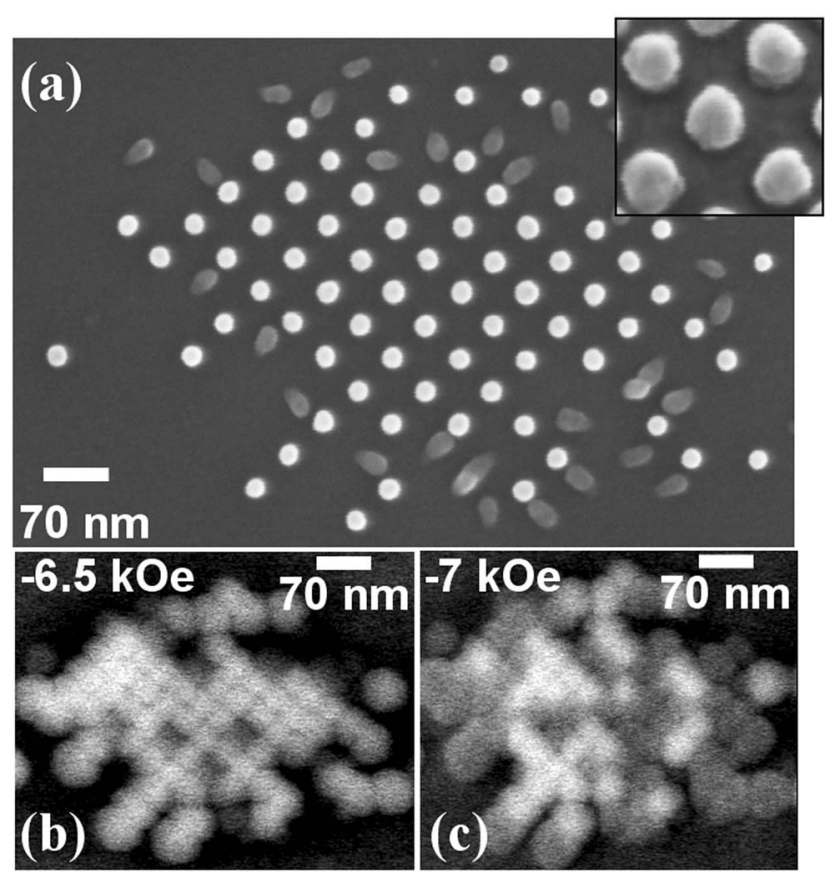

FIG. 2. (a) $\mathrm{SEM}$ image of $\mathrm{SiO}_{x}$ pillars with a period of $50 \mathrm{~nm}$. The inset shows an SEM image after $\mathrm{Co} / \mathrm{Pd}$ multilayer film deposition $\left[d_{\mathrm{tot}}\right.$ $=13.2 \mathrm{~nm}(n=8)]$ with the sample tilted by $15^{\circ}$ revealing the mushroom shape. Corresponding MFM images of magnetic island arrays after applying a $20 \mathrm{kOe}$ vertical field and then reverse fields of (b) $-6.5 \mathrm{kOe}$ and (c) $-7.0 \mathrm{kOe}$, respectively.

In order to create the $\mathrm{SiO}_{x}$ pillar arrays, EUV-IL was performed at the XIL beamline (Swiss Light Source), exposing a $30 \mathrm{~nm}$-thick hydrogen silsesquioxane (HSQ) resist spin-coated on a silicon substrate. The EUV-IL mask comprises four linear gratings fabricated with electron beam lithography. The island array patterns formed by the interfering beams have periods ranging from 100 down to $50 \mathrm{~nm}$, which is smaller than the period of the gratings by a factor of 1.4. ${ }^{10,11}$ The array area for the largest island period of $100 \mathrm{~nm}$ is $500 \times 500 \mu \mathrm{m}^{2}$ and for the smallest island period of $50 \mathrm{~nm}$ studied here is $20 \times 20 \mu \mathrm{m}^{2}$. The gap between mask and substrate is fixed at $160 \mu \mathrm{m}$ to ensure that the maximum interference area is exposed on the resist. Once the HSQ resist has been exposed, it is developed in a tetramethyl ammonium hydroxide solution to give an array of 30 nm-high $\mathrm{SiO}_{x}$ pillars. Figure 1(a) shows a scanning electron microscopy (SEM) image of EUV-IL fabricated islands with a period of $50 \mathrm{~nm}$. The pillars have a negative sidewall profile [see schematic diagram in Fig. 1(a)] due to the strong absorption of EUV light by the HSQ film so that the top receives a higher exposure dose than the bottom. The negative profile means that deposition of magnetic material on the pillar sidewalls is avoided and an array of magnetic islands is created on top of the pillars, which is magnetically decoupled from the magnetic material in the trenches between the pillars [see SEM and schematic in Fig. 1(b)]. The size of the magnetic islands can be tuned by changing the deposited magnetic film thickness and, to some extent, by varying the exposure dose values, which controls the original $\mathrm{SiO}_{x}$ pillar size. As shown in Fig. 1(b), typically the size of the magnetic islands is larger than the original $\mathrm{SiO}_{x}$ pillars. The size distribution of these $50 \mathrm{~nm}$-period magnetic islands over the $20 \times 20 \mu \mathrm{m}^{2}$ area is narrow with a Gaussian sigma $<5 \%$, analyzed from both atomic force microscopy (AFM) images
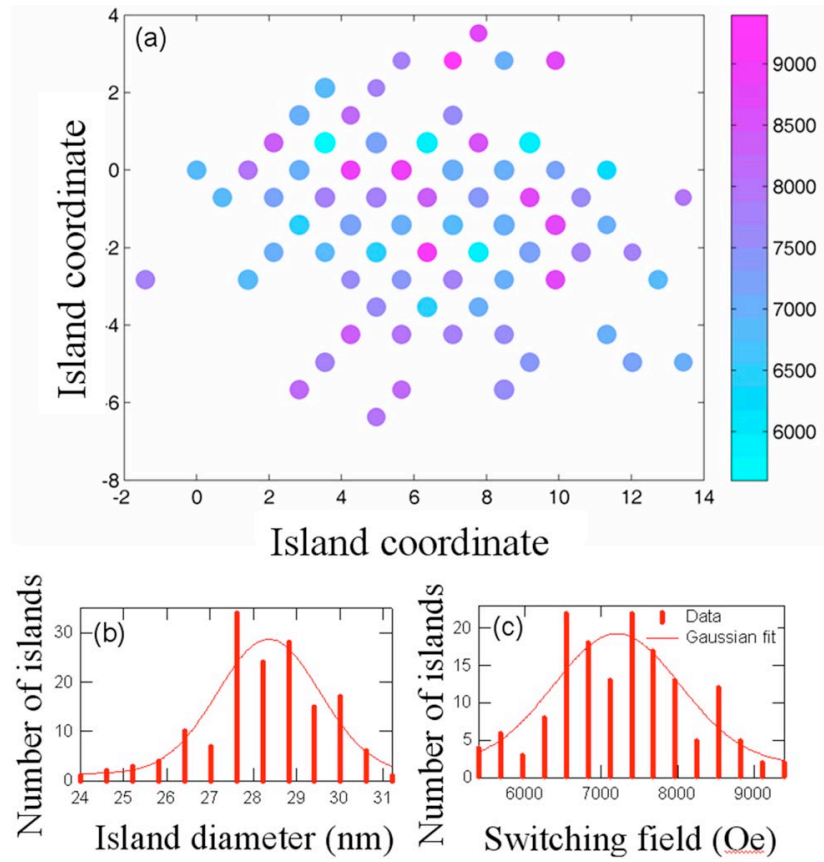

FIG. 3. (Color online) (a) Map of switching fields for each individual island: the color (or grayscale on a black and white printout) corresponds to the switching field and the size is proportional to the magnetic island size for the area shown in Fig. 2(a). (b) The distribution of island diameters $(\sigma /\langle\operatorname{mean}\rangle=5 \%)$ and (c) the distribution of remanent switching fields $(\sigma /\langle$ mean $\rangle=11.5 \%)$. The solid lines are Gaussian fits from which the array mean and sigma are determined.

and the SEM images. The AFM measurements confirmed that the pillar height was $30 \mathrm{~nm}$, consistent with the resist thickness. The $\mathrm{Co} / \mathrm{Pd}$ magnetic multilayer films were deposited by dc magnetron sputtering at room temperature using an Ar pressure of 3 mTorr. The total deposited film consists of a Ta buffer layer (1.2 nm), a Pd (111) textured seed layer $(1.5 \mathrm{~nm})$, a magnetic multilayer $([\mathrm{Co}(0.28 \mathrm{~nm}) /$ $\left.\operatorname{Pd}(0.9 \mathrm{~nm})]_{n}\right)$ with perpendicular anisotropy, where $n$ is the number of layers, and a Pd $(1.1 \mathrm{~nm})$ cap layer to protect against oxidation.

The effect of the size distribution on the SFD was investigated on a $50-\mathrm{nm}$ period array with total film thickness $d_{\mathrm{tot}}=13.2 \mathrm{~nm}(n=8)$. In order to unambiguously identify individual islands, we chose three inhomogeneously exposed areas (comprising a total of 289 magnetic islands) at the edge of a $\mathrm{SiO}_{x}$ pillar array. This allowed easy identification of each island so that it was possible to match each island's switching field measured with magnetic force microscopy (MFM) with the island size measured with high resolution SEM. An SEM image of the $\mathrm{SiO}_{x}$ pillars in one of the three measured areas is shown in Fig. 2(a) where it can be seen that some of the pillars near the edge of the pattern have fallen down. This allows us to directly observe the negative sidewall profile indicated by their triangular shape. The inset of Fig. 2(a) shows the SEM image of pillars after film deposition. The growth of the magnetic film on the $\mathrm{SiO}_{x}$ pillars during deposition results in a mushroom-like structure, also schematically shown in Fig. 1(b). MFM provides a useful local technique to measure the switching of single magnetic islands because of the high spatial resolution $(\sim 20 \mathrm{~nm})$. The MFM measurements were carried out using a Veeco Nanoscope Dimension 3100 with a cobalt-coated high resolution MFM tip (Team Nanotech GmbH). In order to measure the switching field of each magnetic island, we first applied a 
positive $20 \mathrm{kOe}$ magnetic field to the sample to ensure that all of the islands are magnetized in one direction. Then, we applied a reverse field, increasing the field in steps of 200 Oe, imaging the sample at remanence to determine which islands have switched. Two MFM images at applied fields of -6.5 and $-7.0 \mathrm{kOe}$ are shown in Figs. 2(b) and 2(c), which were taken from the same area as the SEM image of the HSQ pillars in Fig. 2(a). The islands have either a dark or a light contrast, implying that each island is a single domain with perpendicular anisotropy and the island is magnetized either parallel or antiparallel, respectively, to the tip field. Figures 2(b) and 2(c) show that as the field is increased from -6.5 to $-7.0 \mathrm{kOe}$, the islands switch independently of their neighbors indicating that there is no exchange coupling between the islands.

The MFM images allow the switching field of each individual magnetic island to be determined and we correlate this with the island size measured by SEM. A map of switching fields for each individual island in Fig. 2(a) is shown in Fig. 3(a): the color corresponds to the switching field and the size is proportional to the magnetic island size determined from the SEM images of the HSQ pillars, taking into account the increase in size due to the overgrowth of the magnetic material. Figures 3(b) and 3(c) show the distribution of island diameter and switching fields for all 289 islands together with Gaussian fits. The mean island diameter is $28.4 \mathrm{~nm}$ after deposition of the magnetic film, which is approximately $7 \mathrm{~nm}$ larger than the original pillar diameter due to the film overgrowth. The Gaussian fit of the size distribution yields $\sigma=1.2 \mathrm{~nm}(\sigma /\langle$ mean $\rangle=5 \%)$, demonstrating the narrow size distribution obtained from EUV interference lithography. The mean switching field is $7200 \mathrm{Oe}$ with $\sigma=830 \mathrm{Oe}(\sigma /\langle$ mean $\rangle=11.5 \%)$ which is comparable with previous results on samples produced by electron beam lithography. ${ }^{9}$

Figure 4(a) is a plot of island switching field as a function of island size. The error bars show the standard deviation of the switching field for each island diameter. Figure 4(b) compares this measured SFD as a function of island size with the simulated SFD due only to the magnetostatic fields from neighboring islands, considering the contribution to the SFD from the 20 nearest neighbor island shells (it should be noted that our calculations have shown that there is little magnetostatic interaction between the magnetic islands and the "antidot film" in the trenches). Also shown is a single data point where the SFD of the entire island population [sigma from Fig. 3(c)] is plotted against the average diameter. The noise on the data arises from the relatively small number of islands at each diameter point. Within the noise limit, the data immediately show that the majority of the SFD is not due to magnetostatic interactions since the ratio of magnetostatic to measured SFD is approximately 0.2-0.3, in agreement with the findings of Hellwig et al. ${ }^{9}$ This can also be seen directly by examining Fig. 3(a) where the immediate surroundings of an individual island do not significantly affect its switching field. The simulations for the magnetostatic SFD show an increase in SFD as the island size increases, as expected for an array with a fixed period $(p=50 \mathrm{~nm})$. Within the noise base of these measurements, we do not observe a strong dependence of the SFD on island size. The relatively small contribution of the magnetostatic environment to the SFD implies that a distribution of mate-

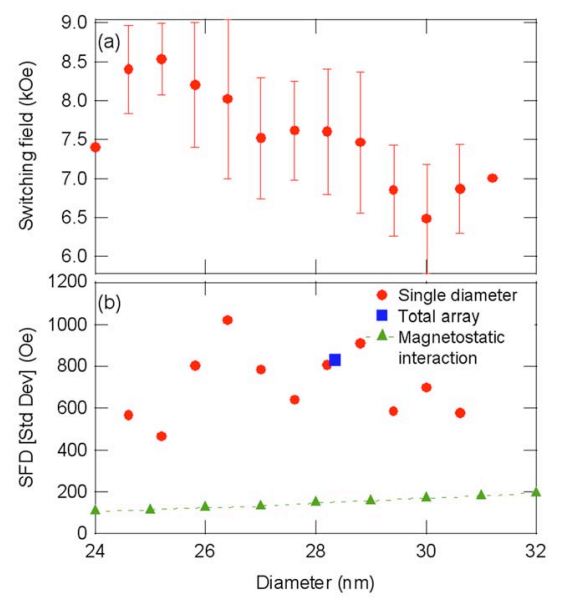

FIG. 4. (Color online) (a) Switching field as a function of island diameter. The error bar is the standard deviation of the switching field for each island diameter. (b) Comparison of the measured SFD as a function of island diameter (circles) with the simulated SFD (triangles) assuming only magnetostatic interactions from neighboring islands contributes to the SFD. The single data point (square) is the SFD of the entire island population.

rial properties such as anisotropy is responsible for the SFD, in agreement with previous work. ${ }^{8}$

In conclusion, we have fabricated perpendicular nanoscale magnetic island arrays with mean island size of $28.4 \mathrm{~nm}$ and $\sigma=5 \%$ on prepatterned substrates with periods of $50 \mathrm{~nm}$ by EUV-IL. SEM and MFM measurements on the same islands have allowed direct comparison of the size and switching field. The results indicate that the SFD is dominated by a distribution of the material properties, and hence by improving the homogeneity of the material, the SFD could be further decreased. In addition, recent advances in EUV-IL have demonstrated that periods of $25 \mathrm{~nm}$ are achievable, ${ }^{13}$ providing a competitive technology for fabrication of future data storage devices at $1 \mathrm{Tbit} / \mathrm{in}^{2}$.

The authors would like to thank Dr. Olav Hellwig and Dr. Yasin Ekinci for their help with sample fabrication. Part of this work was performed at the SLS, Paul Scherrer Institut, Villigen, Switzerland. Financial support by the European Community through the STREP project MAFIN (No. 026513) is gratefully acknowledged.

${ }^{1}$ C. L. Dennis, R. P. Borges, L. D. Buda, U. Ebels, J. F. Gregg, M. Hehn, E. Jouguelet, K. Ounadjela, I. Petej, I. L. Prejbeanu, and M. J. Thornton, J. Phys.: Condens. Matter 14, R1175 (2002).

${ }^{2}$ B. D. Terris and T. Thomson, J. Phys. D 38, R199 (2005).

${ }^{3}$ S. H. Charap, P. L. Lu, and Y. J. He, IEEE Trans. Magn. 33, 978 (1997).

${ }^{4}$ A. Moser, K. Takano, D. T. Margulies, M. Albrecht, Y. Sonobe, Y. Ikeda, S. H. Sun, and E. E. Fullerton, J. Phys. D 35, R157 (2002).

${ }^{5}$ M. Todorovic, S. Schultz, J. Wong, and A. Scherer, Appl. Phys. Lett. 74, 2516 (1999).

${ }^{6}$ H. J. Richter, A. Y. Dobin, R. T. Lynch, D. Weller, R. M. Brockie, O. Heinonen, K. Z. Gao, J. Xue, R. J. M. van de Veerdonk, P. Asselin, and M. F. Erden, Appl. Phys. Lett. 88, 222512 (2006).

${ }^{7}$ R. P. Cowburn, D. K. Koltsov, A. O. Adeyeye, and M. E. Welland, J. Appl. Phys. 87, 7067 (2000).

${ }^{8}$ T. Thomson, G. Hu, and B. D. Terris, Phys. Rev. Lett. 96, 257204 (2006).

${ }^{9}$ O. Hellwig, A. Berger, T. Thomson, E. Dobisz, Z. Z. Bandic, H. Yang,

D. S. Kercher, and E. E. Fullerton, Appl. Phys. Lett. 90, 162516 (2007).

${ }^{10}$ L. J. Heyderman, H. H. Solak, C. David, D. Atkinson, R. P. Cowburn, and F. Nolting, Appl. Phys. Lett. 85, 4989 (2004).

${ }^{11}$ H. H. Solak, J. Phys. D 39, R171 (2006).

${ }^{12}$ G. Hu, T. Thomson, M. Albrecht, M. E. Best, B. D. Terris, C. T. Rettner, S. Raoux, G. M. McClelland, and M. W. Hart, J. Appl. Phys. 95, 7013 (2004).

${ }^{13}$ H. H. Solak and Y. Ekinci, J. Vac. Sci. Technol. B 25, 2123 (2007). 